Research Paper

\title{
Endogenous Estrogen Attenuates Hypoxia-Induced Pulmonary Hypertension by Inhibiting Pulmonary Arte- rial Vasoconstriction and Pulmonary Arterial Smooth Muscle Cells Proliferation
}

\author{
Dunquan $\mathrm{Xu}^{*}$, Wen $\mathrm{Niu}^{*}$, Ying Luo*, Bo Zhang, Manling Liu, Haiying Dong, Yi Liu ${ }^{\bowtie}$, Zhichao Li ${ }^{\bowtie}$ \\ Department of Pathology \& Pathophysiology, Xijing Hospital, Fourth Military Medical University, NO.169 of Changle Western Street, Xi'an, \\ 710032, China. \\ * These authors contributed equally to this study.
}

$\triangle$ Corresponding author: Zhichao Li and Yi Liu, Dept. Pathology \& Pathophysiology, Xijing Hospital, Fourth Military Medical University, NO.169 of Changle Western Street, Xi'an, 710032, China. Phone: +86 029 84774551. Fax: +86 029 84774548. E-mail: lizhic@fmmu.edu.cn, xaliuyi@fmmu.edu.cn.

(C) Ivyspring International Publisher. This is an open-access article distributed under the terms of the Creative Commons License (http://creativecommons.org/ licenses/by-nc-nd/3.0/). Reproduction is permitted for personal, noncommercial use, provided that the article is in whole, unmodified, and properly cited.

Received: 2013.01.18; Accepted: 2013.04.15; Published: 2013.04.22

\begin{abstract}
Exogenous estrogen was shown to exert various beneficial effects on multiple diseases including hypoxia-induced pulmonary hypertension (HPH). However, the effect of endogenous estrogen on $\mathrm{HPH}$ was seldom investigated. In the present study, we explored the protective effects and mechanisms of endogenous estrogen on hypoxia-induced pulmonary hypertension. Male, female, pregnant and ovariectomized rats were housed in a hypoxic condition for 21 days, and then hemodynamic together with morphologic indexes of pulmonary circulation were measured. The right ventricular systolic pressure, mean pulmonary artery pressure, right ventricular hypertrophy index, and arterial remodeling index were significantly elevated after chronic hypoxia exposure. Experimental data showed less severity in female, especially in pregnant rats. In vitro, artery rings of different sex or estrus cycle rats were obtained, and then artery rings experiments were performed to investigate pulmonary vasoconstriction by recording the maximum phase II vasoconstriction. Data showed that the vasoconstriction was milder in proestrus female than diestrus female or male groups, which could be leveled by treating U0I26 (a MAPK pathway inhibitor). Pulmonary arterial smooth muscle cells isolated from different sex or estrus cycle rats were cultured in the condition of $2 \%$ oxygen for 24 hours, and cell proliferation was evaluated by the $[3 \mathrm{H}]$-thymidine incorporation assay. Cells from proestrus rats exhibited lower proliferation than the other groups, which could be countered by both U0I 26 and raloxifene (a selective estrogen receptor modulator). Serum estradiol levels were detected, and rats with higher levels showed less severity of pulmonary hypertension. Conclusively, endogenous estrogen may alleviate hypoxia-induced pulmonary hypertension by attenuating vasoconstriction through non-genomic mechanisms and inhibiting smooth muscle cells proliferation through both genomic and non-genomic mechanisms.
\end{abstract}

Key words: Endogenous estrogen; hypoxia-induced pulmonary hypertension; pulmonary vascular remodeling; hypoxic pulmonary vasoconstriction; estrous cycle.

\section{Introduction}

According to the guideline for the diagnosis and treatment of pulmonary hypertension, lung disease and/or hypoxia-induced pulmonary hypertension $(\mathrm{HPH})$ is a subset of total five classifications [1]. Lung 
diseases related hypoxia or chronic exposure to high altitude could result in $\mathrm{HPH}$. As the other types of pulmonary hypertension, $\mathrm{HPH}$ is also characterized by sustained elevation of pulmonary arterial pressure and vascular resistance [2]. The pathogenesis of $\mathrm{HPH}$ is regarded as hypoxic pulmonary vasoconstriction (HPV) and hypoxia-induced pulmonary vascular remodeling (PVR). Chronic hypoxia can elicit vasoconstriction secondary to increase in circulating vasoconstrictor agonists such as angiotensin, endothelin-1, serotonin, and others, and further resulting in exaggerated PVR [3-5]. Initially, HPV is a compensation for oxygen shortening. After overcompensation, pulmonary vessels response to hypoxia will finally result in $\mathrm{HPH}$, during which the endogenous agonists will be further exaggerated. PVR is a vascular reaction to the elevated pulmonary pressure and vascular resistance due to hypoxia, which in return gears up pulmonary pressure and vascular resistance, thereby a vicious circle forming. Continuing hypoxia eventually causes more severe PVR which can hardly be reversed, and forms the main pathological changes in $\mathrm{HPH}$.

Studies have shown that estrogen could attenuate $\mathrm{HPH}$ through different pathways [6-9]. Besides the classic nuclear-initiated (or genomic) pathway through which estrogen exerts its protective effects by estrogen receptor $\alpha$ or $\beta$, there is a membrane-initiated (or non-genomic) pathway which mediated by a $G$ protein-coupled receptor 30 (GPR30) [10]. The genomic mechanisms of estrogen rely on new proteins-production to mediate its effects, and consequently, such effects are characterized by delayed in onset and prolonged in duration. On the contrary, the non-genomic effects occur rapidly in seconds to minutes, and depend on existing proteins or other molecules such as intracellular calcium for effects [11]. In the present study, we investigated through which ways the endogenous estrogen protects the pulmonary circulation.

Generally speaking, significant sex differences exist in patients suffered from pulmonary hypertension. Usually, females are much more susceptible than males to the primary pulmonary hypertension [2]. However, there were reports that the prevalence is higher in men than women on HPH due to high altitude, and the symptoms of HPH are less serious in women [12-14]. Though studies on sex difference of HPH were carried out early in 1966[12], the exact mechanisms underlying are not clearly elucidated till today.

Studies of endogenous estrogen demonstrated the sex difference in estrogen levels. Additionally, notable difference of endogenous estrogen exists in female menstrual cycle, and estrogen level is elevated remarkably in pregnant phase. Experiments have well elucidated that estrogen level significantly decreases in ovariectomized female animals. Sex difference in estrogen expression gives rise to notably different prevalence of various cardiovascular diseases in human. Endogenous estrogen has diverse effects on cardiovascular system including decreasing vasoreactivity, alleviating hypoxic pulmonary vasoconstriction, and inhibiting cell mitosis in proliferating diseases such as atherosclerosis.

To date, there are few reports about sex differences in development of HPH, and even less discussed the body estrogen levels and HPH severity. Dr. Resta et al. found that ovariectomized rats developed more severe PVR and higher right ventricular hypertrophy index (RVHI) than rats with intact ovaries under chronic hypoxia exposure [7]. Lahm and colleagues further demonstrated endogenous estrogen effects on the pulmonary artery vasoreactivity and acute hypoxic pulmonary vasoconstriction [15]. They found that animals in proestrus, known to have physiologically increased estrogen levels, exhibited an attenuated response to vasoconstrictor compared with estrus, diestrus, and male animals. Although the aforementioned documents unfold a promising picture before us, the exact mechanisms beneath the phenomena are not fully explored. Herein, we hypothesized that endogenous estrogen could have beneficial effects on $\mathrm{HPH}$. In the present study, we established HPH models of different sexual, naturally pregnant, and artificially ovariectomized rats. We further observed hypoxia-induced vasoconstriction and pulmonary arterial smooth muscle cell proliferation.

\section{Materials and Methods}

\section{Animals}

Adult Sprague-Dawley rats (250-350 g) were purchased from the animal center of the Fourth Military Medical University (Xi'an, Shaanxi, China). All the protocols and surgical procedures adopted in this study were reviewed and approved by the Animal Care and Use Committee of the Fourth Military Medical University (approval ID fmmu-11-5078), and complied with the Declaration of the National Institutes of Health Guide for Care and Use of Laboratory Animals (Publication No. 85-23, revised 1985).

\section{In Vivo experiments: duplicate mimetic hy- poxia-induced pulmonary hypertension rat models}

Aiming to explore the effects of endogenous estrogen on hypoxic pulmonary hypertension, adult male or female rats were randomly divided into 8 
groups $(n=5)$ : normoxic male $(\mathrm{NM})$, hypoxic male (HM), normoxic female (NF), hypoxic female (HF), normoxic pregnant female (NP), hypoxic pregnant female (HP), normoxic ovariectomized female (NO), and hypoxic ovariectomized female $(\mathrm{HO})$. The pregnant status was determined at first day of fertilization by vaginal smears method as described [16].

Animals of hypoxic groups were housed in a hypobaric hypoxia chamber depressurized to 380 $\mathrm{mmHg}$ (Correspondingly, PO2 was reduced to about $79.6 \mathrm{mmHg}$ ) for continuing 21 days. The normoxic groups were housed at ambient barometric pressure (about $718 \mathrm{mmHg}, \mathrm{PO} 2$ is about $150.6 \mathrm{mmHg}$ ). All animals were maintained in a 12:12-hour's light-dark cycle condition, and were allowed ad libitum access to food and water. The padding stuff for the animals was changed once a week. The room temperature was air-conditioned at $25^{\circ} \mathrm{C}$.

\section{Hemodynamic analysis and tissue preparation}

After 21 days hypoxia exposure, the animals were anesthetized with $20 \%$ ethylurethanm $(4 \mathrm{~mL} / \mathrm{kg}$ i.p.; Sigma-Aldrich CO. LLC, MO, USA), and a special self-made silicagel catheter linked to the Powerlab system (AD Instruments, Bella Vista, NSW, Australia) was inserted into the pulmonary artery through right jugular vein. There would emerge peculiar waves when the catheter entered the right ventricle and pulmonary artery. The right ventricle systolic pressure (RVSP) and the mean pulmonary arterial pressure (mPAP) were then recorded. At the meantime, the mean carotid artery pressure (mCAP) was also recorded via a special plastic catheter inserted into the carotid artery. After the hemodynamic data were recorded, blood samples were collected from the right jugular vein. The samples were kept at $4{ }^{\circ} \mathrm{C}$ for half an hour, and then centrifuged at $1000 \mathrm{rpm}$ for 5 minutes. Serum was carefully pipetted and stored at $-20^{\circ} \mathrm{C}$ for the next hormone assays.

Sternotomy surgery was performed after obtained the blood samples. After perfused by paraformaldehyde, lungs together with heart were harvested en bloc. The weight of right ventricle (RV) and left ventricle plus septum $(\mathrm{LV}+\mathrm{S})$ were obtained, and the ratio of $(\mathrm{RV} / \mathrm{LV}+\mathrm{S})$ was calculated as the right ventricle hypertrophy index (RVHI). The lungs were sectioned into 4-mm-thick slices at the same part (the lower lobe of the right lung) and soaked in 10\% formalin solution ( $\mathrm{PH}=7.4)$.

\section{Pathomorphologic analysis}

After fixed in $10 \%$ formalin for 72 hours, the lung slices were embedded in paraffin and sectioned into 4 - $\mu \mathrm{m}$-thick sections. To detect the vascular changes in pulmonary arterioles, immunohistochemistry for a-smooth muscle actin (1:800, Millipore, Bedford, MA, USA) was done. The stained lung sections were processed in a double blind method by pathologists for morphological analysis. Pulmonary arteries which external diameter ranged from 50 to $200 \mu \mathrm{m}, 6-10$ vessels with approximate round shape were obtained from every rat; total 40 arteries were got from each group. The average size of the obtained vessels was 75 $\mu \mathrm{m}$. The external and internal diameters of pulmonary arterioles were measured by an image-processing program (Image-Pro Plus, Version 5.1, Media Cybernetics, Bethesda, MD, USA). The cross sectional area of medial wall and the total cross sectional vessel area were then obtained. Pulmonary vascular structure remodeling was assessed by WA \% $=($ wall area $) /($ vessel cross sectional area $) \times 100 \%$.

\section{Pulmonary arteries vasoconstriction experi- ments}

To explore the effects of endogenous estrogen on the vasoconstriction and vasoreactivity, pulmonary arteries obtained from adult Sprague-Dawley rats were grouped as follow $(n=10)$ : male $(M)$, diestrus female (DE), proestrus female (PE), proestrus female treated with raloxifene (Sigma-Aldrich CO. LLC, MO, USA) (PE+R), proestrus female treated with 1,4-Diamino-2,3-dicyano-1,4-bis(o-aminophenylmerc apto) butadiene monoethanolate (U0126, Sigma-Aldrich CO. LLC, MO, USA) (PE+U), and proestrus female group treated with both raloxifene and U0126 (PE+R\&U). The menstrual cycle of the female rats was determined by vaginal smears method. Briefly, examined by microscopy, most of the cells on the smear are white cells with a few of epithelial cells in the diestrus phase. While in the proestrus phase, most of the cells on the smear are inflated oval epithelial cells. When in the estrus phase, the inflated epithelial cells disappear and turn into squamous keratinized epithelial cells which pile up like piles of leaves.

Rats were anesthetized with intraperitoneal injections of $20 \%$ ethylurethanm $(4 \mathrm{~mL} / \mathrm{kg})$. Serum samples were collected as the procedure mentioned above and stored in $-20^{\circ} \mathrm{C}$ for next hormone analysis. Median sternotomy was performed, and the lungs together with heart were removed into a culture plate with $4^{\circ} \mathrm{C}$ oxygenated Krebs-Henseleit $(\mathrm{KH})$ solution (containing: $\mathrm{NaCl} 127, \mathrm{KCl} 4.7, \mathrm{NaHCO}_{3} 17, \mathrm{MgSO}_{4}$ 1.17, $\mathrm{KH}_{2} \mathrm{PO}_{4} 1.18, \mathrm{CaCl}_{2} 2.5$, and D-glucose 5.5, all units are $\mathrm{mM}$.). The third-division (external diameter $<300 \mu \mathrm{m}$ ) pulmonary arteries were carefully isolated and cleared surrounding tissue under a dissecting microscope. The isolated pulmonary arteries were cut into 3-mm-length rings and suspended on steel hooks connected to force transducers (AD Instruments, Bella 
Vista, NSW, Australia) for isometric force measurement. PA rings were immersed in glass waterbaths containing $\mathrm{KH}$ solution continuing bubbled with $95 \%$ $\mathrm{O}_{2}-5 \% \mathrm{CO}_{2}$ at $37{ }^{\circ} \mathrm{C}$. Force data was recorded using the PowerLab system.

The PA rings were stretched to a predetermined optimal passive tension of $750 \mathrm{mg}$. Then the rings were equilibrated for an hour, and the $\mathrm{KH}$ solution was changed every 15 minutes. The viability of PA rings was determined by measuring maximum contractile response to $1 \mu \mathrm{M}$ phenylephrine (PE). Endothelium integrity of each artery ring was determined by demonstrating whether acetylcholine (Ach)-induced relaxation of intact pulmonary vessel precontracted with phenylephrine was adequate. Total 10 pulmonary arteries were obtained in each group. After Ach being washed out, PA rings were equilibrated for an hour. Following equilibration, PA rings were precontracted with phenylephrine again. According to our preliminary experiments (please see the supplemental data), the appropriate concentrations of estrogen-receptor modulator raloxifene (3 $\mathrm{nM}$ ) and MAPK signaling pathway blocker U0126 (50 $\mu \mathrm{M})$ were added in. The bubbled gas was replaced by $95 \% \mathrm{~N}_{2}-5 \% \mathrm{CO}_{2}$ to producing a hypoxic condition for an hour. As figure 1 showed, hypoxia caused a biphasic PA vasoconstriction: an early contraction (appearing at 2-3 minutes after exposure to hypoxia) followed by a transient vasodilation, then delayed sustained phase II (appearing at 15-20 min after hypoxia exposure) contraction. Maximum phase II vasoconstriction was measured as the difference between the highest and lowest force displacements during hypoxia and expressed as a percentage of maximum phenylephrine precontraction.

\section{Pulmonary arterial smooth muscle cells cul- ture and cell proliferation assays}

To study the effects of endogenous estrogen on cell proliferation, rat PASMCs were isolated from male, diestrus female and proestrus females rats, respectively. Cells were obtained by explants method as described previously [8]. The pulmonary arterial explants were cultured in six wells cell-culturing Petri dishes (Jet Biofil Inc, Guangzhou, Guangdong, China), and maintained at $37^{\circ} \mathrm{C}$ in a humidified $5 \% \mathrm{CO}_{2} / 95 \%$ $\mathrm{O}_{2}$ atmosphere. When the cells grew to about $80 \%$ area of the culture dish, then the explants were removed and the primary cultured cells were used in next experiments. Each group of PASMCs was identified by immunocytochemical staining for a-smooth muscle actin before proliferation assay. Usually, the purity of primary cultured PASMCs is $90-95 \%$. Cells were grouped according to vasoconstriction assay.

PASMC proliferation was evaluated using the $\left[{ }^{3} \mathrm{H}\right]$-thymidine incorporation assay. When the PASMCs were observed to grow to about $80 \%$ percent area of the culturing plate, the explants were removed, the medium was substituted with phenol-red-free DMEM/F12 containing 0.1\% FBS. After serum starvation for 24 hours, $\left[{ }^{3} \mathrm{H}\right]$-thymidine $(0.4$ $\mu \mathrm{Ci} / \mathrm{mL}$, Amersham, Uppsala, Sweden) was added in with absence or presence of raloxifene $(3 \mathrm{nM})$ and U0126 $(50 \mu \mathrm{M})$, and then cells were removed into a $2 \%$ oxygen cell incubator (HERAcell 240, Heraeus Inc., Hanau, Germany) for another 24 hours. After the incubation, cells were removed to a $10 \mathrm{~mL}$ centrifuge tube, washed twice with cold Hank's balanced salt solution, fixed with ice-cold methanol and precipitated by $10 \%$ trichloroacetic acid. At last, sedimentation samples were lysed in $0.1 \mathrm{M} \mathrm{NaOH}$ solution, transferred to four $\mathrm{mL}$ of scintillation solution and counted by a beta-counter (LabLogic Systems Inc. Brandon, FL, USA) to determine CPM values. Each group was performed on quintuple cultures and repeated 3 times independently. The proliferation of PASMCs is shown as a percentage of CPM after hypoxia treatment to the CPM of unstimulated PASMCs under $0.1 \%$ FCS.

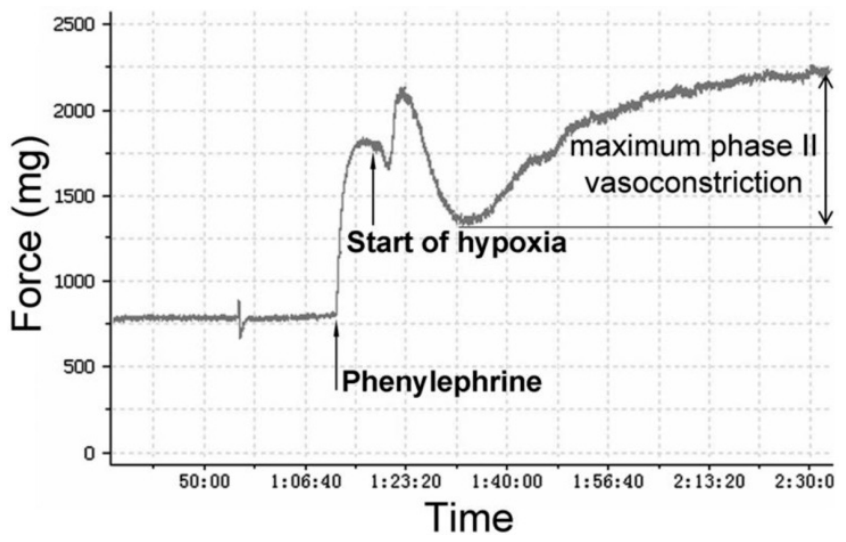

Fig I. Pressure tracing of hypoxic vasoconstriction in isolated pulmonary arteries (PAs). PAs precontracted using phenylephrine (I $\mu \mathrm{M} / \mathrm{L})$ were exposed to hypoxia $\left(\mathrm{PO}_{2} 30-35 \mathrm{mmHg}\right)$ for $60 \mathrm{~min}$. Maximum phase II vasoconstriction was measured as the difference between the lowest force preceding contraction and the highest force during $60 \mathrm{~min}$ of hypoxia.

\section{Hormone assays}

To investigate the difference of hormone levels among all groups mentioned above (those were: NM, $\mathrm{HM}, \mathrm{NF}, \mathrm{HF}, \mathrm{NP}, \mathrm{HP}, \mathrm{NO}, \mathrm{HO}, \mathrm{PE}$ and DE total ten groups), a commercially available radioimmunoassay kit (Biomerica, Newport Beach, CA, US) was employed. All the blood samples were collected from the right jugular vein and detected according kit protocol. Briefly, $25 \mu \mathrm{L}$ of each serum sample was added to each of two glass tubes, together with a known amount of radioactive estradiol and anti-estradiol 
antibody. After the tubes being incubated at $37^{\circ} \mathrm{C}$ for $90 \mathrm{~min}$, a precipitant solution was added to remove the antibody (and bound estradiol) from solution. Tubes were centrifuged for $20 \mathrm{~min}\left(4^{\circ} \mathrm{C}, 3000 \mathrm{rpm}\right)$, the supernatant was discarded and the radioactivity of the remaining pellets was measured by using a gamma counter. A standard curve was created by adding known amounts of estradiol to tubes, and then the concentration of each experimental sample was obtained. The detection limit of this assay was 7.2 $\mathrm{pg} / \mathrm{mL}$.

\section{Statistical analyses}

All values were expressed as mean \pm SD. Statistical analysis was processed by using one-way ANOVA, followed by LSD test for post hoc multiple comparisons (SPSS for Windows version 16.0, Chicago, IL, USA). Significant difference was accepted at $P$ $<0.05$.

\section{Results}

\section{Pulmonary hypertension severity of different sex, pregnant and ovariectomized rats under chronic hypoxia}

After 21 days hypoxia exposure, the RVSP of hypoxic groups were significantly elevated compared with normoxic groups (Figure 2a). In the HF and HP groups, RVSP were significantly lower than those in the $\mathrm{HM}$ and $\mathrm{HO}$ groups $(\mathrm{P}<0.05$, Figure $2 \mathrm{a})$. However, there was no significant difference between $\mathrm{HO}$ and HM groups (Figure 1a). Additionally, though the RVSP of HP group was lower than that of the HF group, no significant difference was found between them (Figure 2a). The pressure was nearly equal between male and female under normoxic exposure. The variation tendency of mPAP among every group was similar as RVSP. Compared with normoxia, hypoxia notably increased mPAP $(P<0.05$, Figure $2 b)$. Compared with male in the same hypoxic condition, female showed significant lower $(P<0.05$, Figure $2 b)$ and pregnant rats exhibited lowest $\mathrm{mPAP}$, but ovariectomized female presented no significant difference (Figure 2b). No significant difference was found between each normoxic group (Figure $2 b$ ). In addition, there was no distinct difference of the mCAP between each group (Figure 2c).

As the data showed (Figure 3), thickened pulmonary arterial adventitia and accumulated extracellular matrix were observed in the lung sections of chronically hypoxic rats (Figure 3B, D, F, H). Obviously, wall-thickened pulmonary arterioles with proliferated and hypertrophic smooth muscle cells, together with inflammatory cells infiltration were seen in the lung of hypoxic rats. Additionally, intima hy- perplasia and lung alveolar structure destructions were shown in hypoxic groups. However, for female and pregnant rats (Figure 3D, F), those vessel changes were much better than male or ovariectomized female (Figure 3B, H) after chronic hypoxia. The changes mentioned above were not observed in the normoxic groups (Figure 3A, C, E, G). Statistically, compared to normoxia, the pulmonary arterial structure remodeling index (WA\%) and right ventricle hypertrophy index (RVHI) were both significantly elevated after chronic hypoxia exposure $(P<0.05$, Figure $3 a$ and $3 b)$. Similarly in hypoxic condition, female and pregnant rats showed relatively less severity on the both indexes (Figure $3 a$ and $3 b$ ).
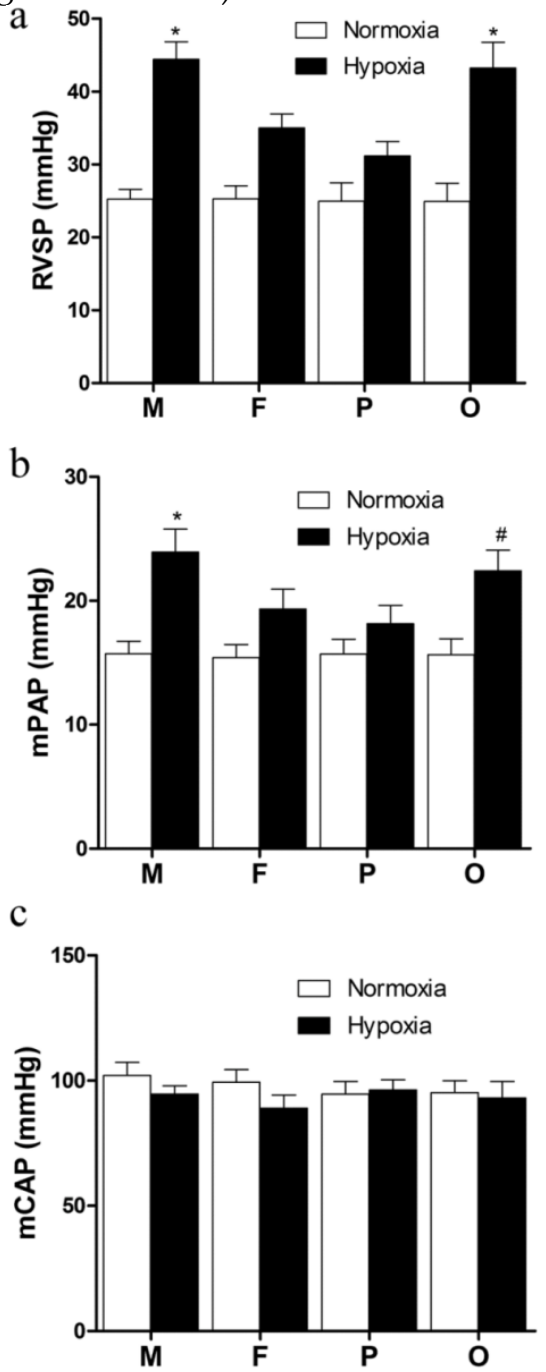

Fig 2. Hemodynamic indexes of different sex, pregnant and ovariectomized rats under normoxia or chronic hypoxia. (a) Right ventricular systolic pressure, ${ }^{*} P<0.05$ vs. hypoxic female group and pregnant group. (b) Mean pulmonary arterial pressure, $* P<0.05$ vs hypoxic female group and pregnant group. ${ }^{\# P}<0.05$ vs. hypoxic pregnant group. (c) Mean carotid arterial pressure. All values are expressed as mean $\pm S D, n=5$. RVSP: right ventricle systolic pressure, mPAP: mean pulmonary arterial pressure, mCAP: mean carotid artery pressure. NM: normoxic male NF: normoxic female, HM: hypoxic male, HF: hypoxic female, NP: normoxic prganancy, HP: hypoxic pregnancy, NO: normoxic ovariectomized female, HO: hypoxic ovariectomized female. 


\section{Effects of estrous cycles and estrogen pathway inhibitors on hypoxia-induced pulmonary vas- oconstriction}

Maximum phase II vasoconstriction of pulmonary artery rings was detected to quantify the vasoconstriction response to hypoxia. As the data showed, the vessels from proestrus female rats had evidently smaller constriction than those from diestrus female or male rats $(P<0.05$, Figure 4$)$. Treated with raloxifene, a selective estrogen receptor modulator alone, the vessels of proestrus female rat showed no significant difference of vasoconstriction compared with untreated ones (Figure 4). While treated with U0126, a MAPK pathway inhibitor, or both raloxifene and U0126, the effect of anti-hypoxia-induced vasoconstriction in proestrus female rats' vessels was completely relieved $(P<0.05$, compared with no treated ones, Figure 4). Therefore, there was no significant difference among the vessels from male, diestrus female, proestrus female rats treated with $\mathrm{U} 0126$ or both
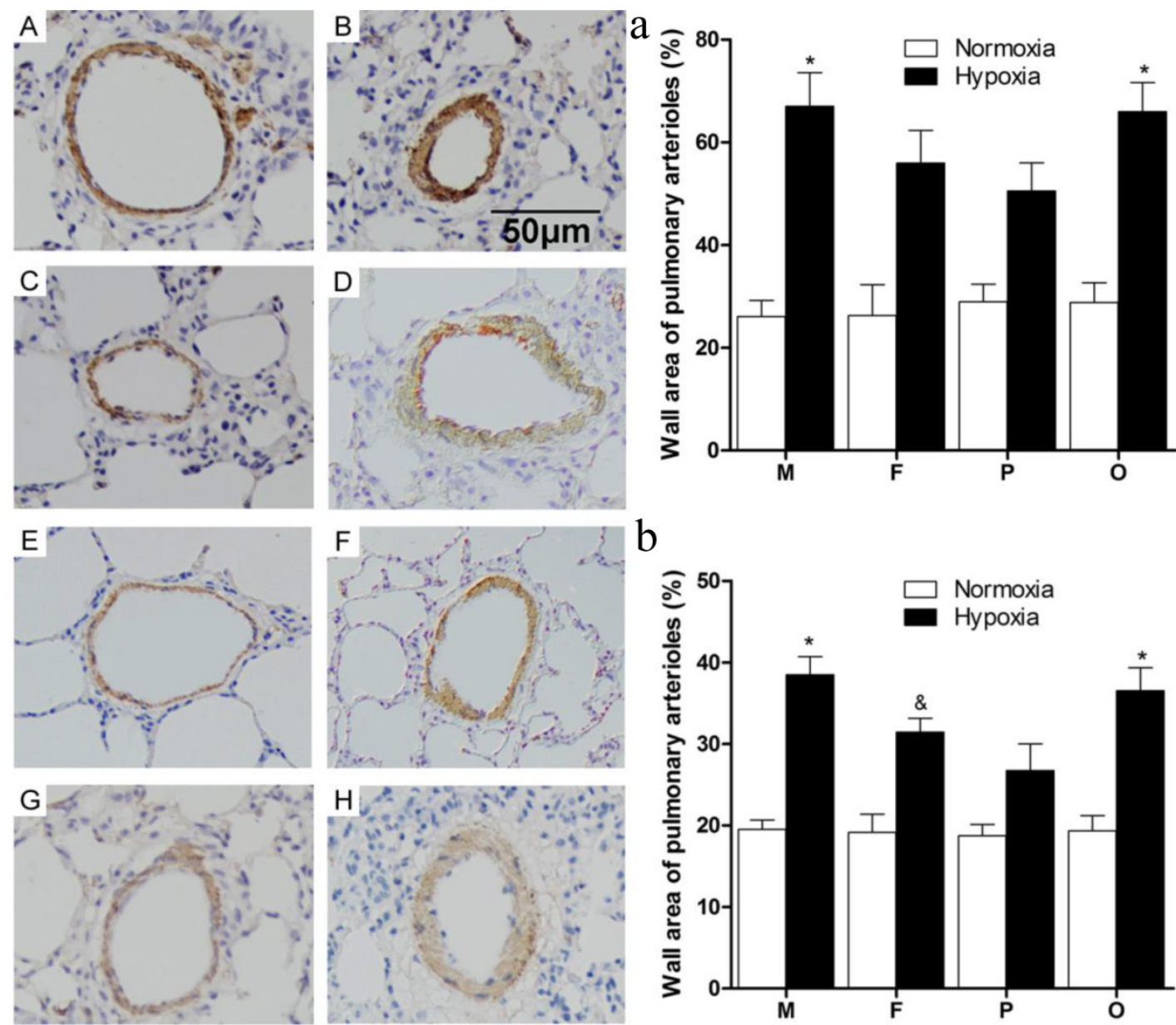

Fig 3. Pulmonary arterioles morphologic changes of different sex, pregnant and ovariectomized rats under normoxia or chronic hypoxia. (A-H) Immunohistochemistry for $\alpha$-smooth muscle actin of pulmonary arterioles (original magnification $\times 20$ ). Scale bars $=50 \mu m$. (a) Wall area of pulmonary arterioles (WA\%). ${ }^{*}<0.05$ vs. hypoxic pregnant group. Arterioles of external diameter ranged from $50-200 \mu m$ were obtained, $n=40$. (b) Right ventricular hypertrophy index $(\mathrm{RVHI}) .{ }^{*} P<0.05$ vs. hypoxic female group and hypoxic pregnant group. \& $P<0.05$ vs. hypoxic pregnant group. All values are expressed as mean \pm SD, $n=5$. NM: normoxic male, HM: hypoxic male, NF: normoxic female, HF: hypoxic female, NP: normoxic pregnancy, HP: hypoxic pregnancy, NO: normoxic ovariectomized female, HO: hypoxic ovariectomized female. A: NM group, B: HM group, C: NF group, D: HF group, E: NP group, F: HP group, G: NO group, $\mathrm{H}$ : $\mathrm{HO}$ group. 


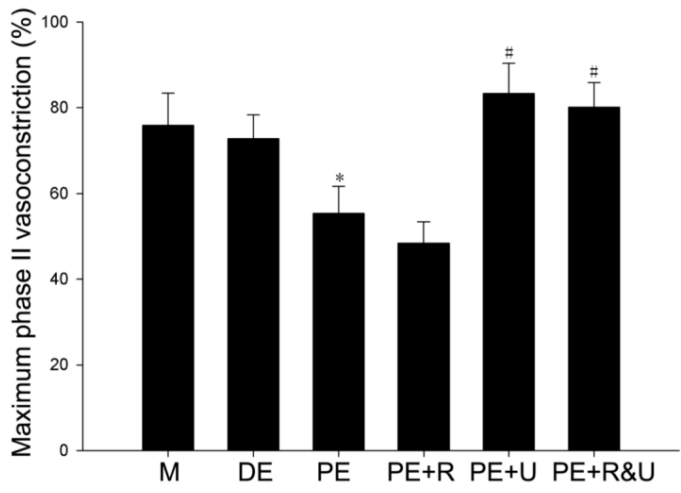

Figure 4. Effects of estrous cycles and estrogen pathway inhibitors on maximum phase II vasoconstriction of pulmonary artery rings induced by hypoxia. ${ }^{*} P<0.05$ vs. $D E$ group. ${ }^{\#} P<0.05$ vs. $P E$ group. Values are expressed as mean $\pm S D, n=10$. M: vessels from male rats, $D E$ : vessels from diestrus female rats, $P E$ : vessels from proestrus female rats, $P E+R$ : vessels from proestrus rats treated with $3 \mathrm{nM}$ raloxifene, $\mathrm{PE}+\mathrm{U}$ : vesssels from proestrus rats treated with $50 \mu \mathrm{M} U 0 \mathrm{I} 26, \mathrm{PE}+\mathrm{R} \& \mathrm{U}$ : vessels from proestrus rats treated with both $3 \mathrm{nM}$ raloxifene and $50 \mu \mathrm{M} \cup 0 \mathrm{I} 26$.

\section{Estrogen levels assays}

The estradiol level of female rats was significantly higher than that of male rats $(P<0.05$, Figure 6$)$ in both normoxia and hypoxia. However hypoxic exposure itself had no effects on animals' serum estradiol level (Figure 6). The estradiol level of pregnant rats was remarkably increased, which was nearly three times than those of no pregnancy ones. But compared with intact ovary female, the ovariectomized rats showed reduced serum estradiol (Figure 6) to a male level. For female rats, estradiol level was a detectable fluctuation during the estrous cycle. The serum estradiol concentration in proestrus was almost 1.7 times higher than that in diestrus (Figure 6).

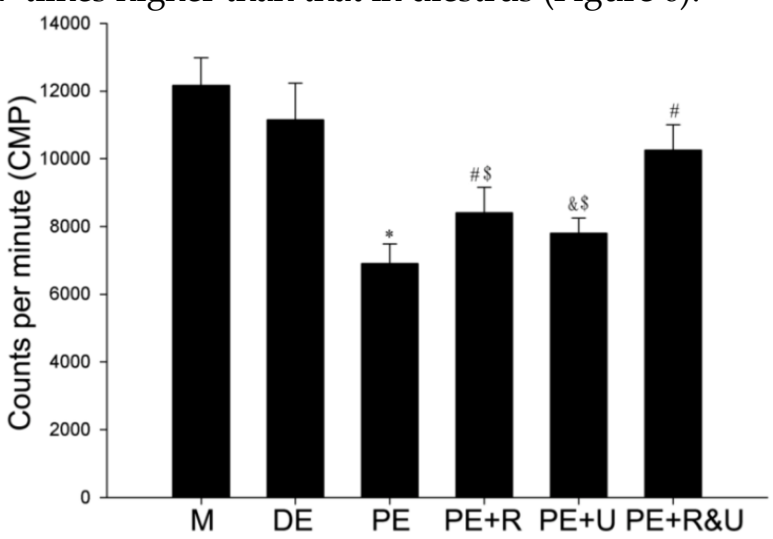

Figure 5. Effects of estrous cycles and estrogen pathway inhibitors on PASMCs proliferation induced by hypoxia. The CPM values are representative of primary cultured PASMCs proliferation. " $P<0.05$ vs. DE group; ${ }^{\#} P<0.01$, ${ }^{\&} P<0.05$ vs. $P E$ group; ${ }^{\$} P<0.05$ vs. $P E$ and $P E+R \& U$ groups. Values are expressed as mean $\pm S D, n=10$. M: cells from male rats, $D E$ : cells from diestrus female rats, $P E$ : cells from proestrus female rats, $P E+R$ : cells from proestrus rats treated with raloxifene, $\mathrm{PE}+\mathrm{U}$ : cells from proestrus rats treated with U0I26, PE+R\&U: cells from proestrus rats treated with both raloxifene and U0I26. The assays were performed on quintuple cultures for each group and repeated for 3 times independently.

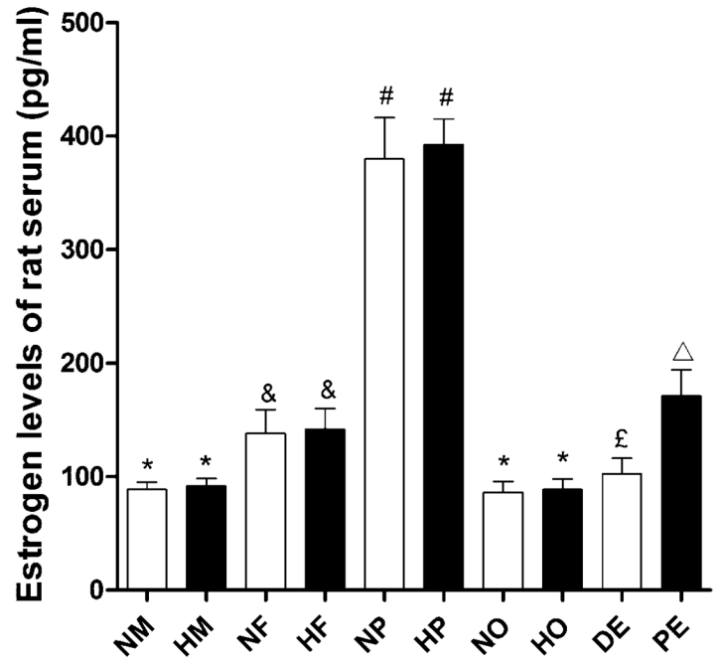

Figure 6. Serum estradiol levels of different sex and estrus, pregnant, ovariectomized rats under normoxia or chronic hypoxia. $P<0.05$ vs. NF, $\mathrm{HF}, \mathrm{NP}, \mathrm{HP}$, and PE groups; ${ }^{\&} \mathrm{P}<0.05$ vs. NM, HM, NP, HP, NO, and HO groups; ${ }^{\# P}<0.05$ vs. NM, HM, NF, HF, NO, HO, DE, and PE groups; ${ }^{f} P<0.05$ vs. NM, HM, NP, HP, and PE groups; $\triangle P<0.05$ vs. NM, HM, NP, HP, NO, $H O$, and $D E$ groups. Values are expressed as mean $\pm S D, n=5$. NM: normoxic male, HM: hypoxic male, NF: normoxic female, HF: hypoxic female, NP: hypoxic pregnancy, HP: hypoxic pregnancy, NO: hypoxic ovariectomized female, $\mathrm{HO}$ : hypoxic ovariectomized female, DE: diestrus female, PE: proestrus female.

\section{Discussion}

There are increasing researches focused on the effects of endogenous hormones on cardiovascular diseases, in which the levels of sex hormones are varied from patients' sex and age [17-20]. Recently, Lee and colleagues [21] demonstrated that endogenous estrogen level is an indicator of stroke risk in older postmenopausal women, that is, older women with high endogenous estrogen had 2.3-fold greater odds of stroke, especially in those with greater central adiposity. On the other hand, there are many studies have shown that administration of exogenous estrogen could attenuate various stimulator-induced pulmonary hypertension $[6,7,22,23]$. However, there have been few studies regarding the effects of endogenous estrogen on pulmonary hypertension. According to earlier study, Rabinovitch et al. showed that adult female rats developed less severe pulmonary hypertension compared to the adult male or infant rats of both male and female[24]. This study also showed that the lung structural damage of adult female was the more light, which provides a clue that age and sex hormone may affect the development of HPH. In 1994, Sardella et al. found that the ovariectomy aggravated the high-altitude-associated polycythemia, elevated the RVSP, and increased the $\mathrm{RV} / \mathrm{LV}+\mathrm{S}$ ratio [25]. Our results showed that the hypoxia-induced pathological changes of female rats were significantly milder than male rats, and the 
pregnant ones were most slight. However, the ovariectomized female group showed a similar severity as male group. These results suggest that various responses from sex or pregnancy may associate with the different endogenous estrogen levels. Our results were partly consistent with the earlier studies mentioned above. We also considered the other factors influenced endogenous estrogen level, such as pregnancy and estrus circle. Moreover, the relationship between endogenous estrogen concentration and hypoxia-induced vessel responses were not fully explored before.

As we know that hypoxia-induced pulmonary vasoconstriction (HPV) is the main change in the acute stage of HPH. Chronic hypoxia results in pulmonary vascular remodeling (PVR), which is characterized by PASMCs proliferating, pulmonary arterioles thickening, vessel lumen narrowing and pulmonary vascular resistance improving. These pathological changes finally lead to the increase of pulmonary arterial pressure and hardly to be reversed. So we next investigated what role of the endogenous estrogen plays in HPV and PVR.

In this study, we demonstrated that pulmonary arteries (PAs) from animal of high physiological estrogen level present relatively low contractility in response to hypoxic exposure. Not only sex, but estrus circle influences the hypoxia-induced acute response of PAs. We found that PAs from proestrus rats exhibited the lowest contraction to hypoxia in the four estrus circle stages. However, the vessels showed a similar contractibility between diestrus and male rats. Tim Lahm et al. showed in their study that hypoxia induced phase II vasoconstriction was significantly attenuated in PA rings from proestrus rats compared to those of the male ones, which was inconsistent with our data [15]. Different from the study of Tim Lahm mentioned above, we found that there was significant difference between proestrus and diestrus rats of the hypoxia-induced phase II vasoconstriction of PA rings. Additionally, Ketan et al. revealed that there were no sex differences in the response of isolated pulmonary artery rings to acute hypoxia [26]. In their study, they adopted castrated and ovariectomized rat models to explore hormone depletion effects on acute hypoxia-induced vasoconstriction. However, Lahm employed different sex and menstrual cycle animals to investigate the endogenous estrogen effects on response of isolated pulmonary artery rings to acute hypoxia. In our present study, not only sex was considered, but also pregnancy, a physiological period with peak endogenous estrogen concentration, was taken into account. Different animal models or apparatus adopted in the studies may explain these discrepancies.
The exaggerated PASMCs proliferation is either of the essential contributions to PAH. Our study demonstrated that PASMCs from different estrous animals exhibited diverse proliferation responses to hypoxia. PASMCs from PE rats showed less proliferation compared to those of male or DE rats. The results revealed that endogenous estrogen may have attenuated the proliferation response of PASMCs to hypoxia exposure. Previous studies had demonstrated the exogenous estrogen's inhibiting effect on proliferation of many kinds of cells [27, 28]. However, no studies have ever been carried out to explore the endogenous estrogen affecting proliferation of PASMCs under hypoxia exposure.

To reveal the relationship between endogenous estrogen and hypoxia-induced pulmonary hypertension, we detected the estradiol levels of each groups of animals. Through investigating the serum estrogen level of each group every four days, we found that the estrogen level of the female rats was $56.7 \%$ higher than male. In the last nine days, the average estrogen concentration of pregnant rats was 4.2 times as male. However, the estrogen level of ovariectomized rats was as much as that of the male group. Taking the results of in vivo experiments into account, the animals with higher levels of estrogen showed lower RVSP and mPAP, and less severity of pathological changes, and the pregnant ones were most slight. However, the ovariectomized female group with lower estrogen level showed a similar severity as male group. Similarly, in vitro study demonstrated that PASMCs from animals with different endogenous estrogen levels exhibited diverse proliferation responses to hypoxia. Rats of high estrogen expression showed less proliferation compared to those of low estrogen levels. Besides, the PA rings experiments showed that the PAs from proestrus rats with the highest serum estrogen level exhibited the lowest contraction to hypoxia. In summary, these results revealed that endogenous estrogen may have attenuated hypoxia-induced PVR, inhibited PASMCs proliferation, and decreased PA rings contraction response to hypoxia exposure. These results suggest that distinctly different estrogen level of animals may provide a good model for investigating the impact of endogenous estrogen on body physiology or pathology.

Then another interesting question rises, that is, why the isolated arterial rings and cultured PASMCs were still affected by endogenous estrogen? In our opinion, it could be due to the following three reasons. Firstly, the different contribution of estrogen receptors in diverse tissues may explain the question. For example, Lindsey found that there exist GPR30 receptors in intima and media of the aorta[29]. Sec- 
ondly, the isolated tissues or cultured cells from different sex animals can still express or secret different levels of estrogen. Thirdly, the original differences between the PASMCs from different animals may affect their response to hypoxia, which remains to be investigated later. A problem whether paracrine or autocrine pathway can affect the isolated organs or cultured cells remains to be further explored.

In the present study, the effects of endogenous estrogen on hypoxia-induced pulmonary vasoconstriction may be membrane-mediated, because the whole experiments were completed within an hour. However, we did not exclude the factor whether the gene expressions of PAs have been altered originally before they were taken out of the animals, which will affect their vasoreactivity to hypoxia, even if the effects of estrogen are blocked by drugs. Our results also demonstrated that blocked ER pathway with raloxifene did not inhibit the vasodilating effect of endogenous estrogen, on the contrary, promoted it. The results were partially consistent with the recent findings [30], in which the researchers demonstrated that GPR30 mediated vasodilatation effects on isolated mesenteric vessels and aorta by administration of selective agonists G-1 and $\mathrm{E}_{2}$. Raloxifene is an estrogen modulator which could bind with ERs with high affinity, and express an estrogen-agonist or estrogen-antagonist effect depending on the tissue targeted [31]. Then we speculated that the raloxifene administered in our study may play an agonist role on GPR30 of the isolated vessels during hypoxia exposure, and improved the non-genomic effects of endogenous estrogen. To date, there were no commercial antagonists of GPR30, so we employed U0126 as the antagonist to block its downstream MAPK signaling pathway. As the results showed, the vasodilating effect was blocked. Additionally, administration of both raloxifene and U0126 showed a moderate effect of vasodilating. So we furthered verifying the effects of endogenous estrogen on the hypoxia-induced vasoconstriction may be mediated by non-genomic mechanisms. However, it was interesting for us to find that in the hypoxia-induced PASMCs proliferation assays, administrating of raloxifene or U0126 could eliminate the inhibiting effects of endogenous estrogen on hypoxia-induced PASMCs proliferation. Combined application of raloxifene and U0126 had a synergistic effect on eliminating the inhibiting effects of endogenous estrogen aforesaid. The GPR30 may then play a rapid inhibiting role on HPV and a promoting role on hypoxia-induced PASMCs proliferation. Though the long-term effects of raloxifene and U0126 on HPH in vivo were not explored, based on the present results, we speculated that the non-genomic MAPK pathway mediated by GPR30 may participate in the proliferation of PASMCs. Further studies regarding the roles of GPR30 together with the MAPK signaling pathway during HPH should be investigated. Taken together, our results revealed that the inhibiting effects of endogenous estrogen on $\mathrm{HPH}$ may through both genomic (by inhibiting PVR and PASMCs proliferation), and non-genomic (by attenuating HPV and MAPK signaling pathway) mechanisms.

Several cardiovascular diseases associated with menstrual cycle, especially the coronary artery disease [32]. There is an "estrogen withdrawal" phenomenon existing in the postmenstrual women, exhibiting as decreased estrogen levels resulting in vasospasm, which tightly associated with vascular diseases [33]. The aforesaid achievements together with our results may explain the difference of PAH incidence between male and female, and no difference between postmenstrual women and men. Also, our results may explain why females exhibit less severe responses compare to males in high altitude conditions.

Other mechanisms could also explain the inhibitory effects of estrogen on HPV and cell proliferation under hypoxic exposure. Administering estrogen could promote various vasodilatation factors release, such as NO or prostacyclin-2[34]. Moreover, inflammation plays an important role in the development of $\mathrm{HPH}$ [35]; therefore the anti-inflammation effects of estrogen may participate in attenuating $\mathrm{HPH}$.

There were studies showed that long-term application of estrogen could result in multiple side-effects [36, 37]. However, short-term administration of large dosage of estrogen may be applied for treating acute hypoxia-induced hypertension, and may effect on preventing ARDS [38]. Our results may provide new strategies to develop medicines which possess the non-genomic effects of estrogen. These medicines may exert protecting effects on chronic hypoxia-induced pulmonary hypertension.

\section{Conclusion}

Our study demonstrated that endogenous estrogen inhibited chronic hypoxia induced right ventricle hypertrophy, decreased pulmonary arterioles thickening, and alleviated HPH. Our data showed that endogenous estrogen may attenuate HPV through non-genomic mechanisms, and inhibit hypoxia-induced proliferation of PASMCs through both genomic and non-genomic mechanisms. Since we cannot take all the factors into account, there must be inevitable shortages in the present study, even though the present study may offer useful information for understand the pathogenesis and treatment of $\mathrm{HPH}$. Further understanding of the endogenous estrogen effects on hypoxia-induced pulmonary arterial con- 
traction and remodeling may have implications for developing new estrogen analogs to treat $\mathrm{HPH}$.

\section{Abbreviations}

HPH: hypoxia-induced pulmonary hypertension; HPV: hypoxic pulmonary vasoconstriction; PVR: pulmonary vascular remodeling; PASMC: pulmonary arterial smooth muscle cell.

\section{Acknowledgments}

This work was supported by the National Natural Science Foundation of China (grants: 30700965; 81200036). The authors thank reviewers for English language checking and editing of the paper.

\section{Competing Interests}

The authors have declared that no competing interest exists.

\section{References}

1. Galie N, Hoeper MM, Humbert M, Torbicki A, Vachiery JL, Barbera JA, Beghetti M, Corris P, Gaine S, Gibbs JS et al: Guidelines for the diagnosis and treatment of pulmonary hypertension: the Task Force for the Diagnosis and Treatment of Pulmonary Hypertension of the European Society of Cardiology (ESC) and the European Respiratory Society (ERS), endorsed by the International Society of Heart and Lung Transplantation (ISHLT). Eur Heart J 2009; 30(20):2493-2537.

2. Runo JR, Loyd JE: Primary pulmonary hypertension. Lancet 2003; 361(9368):1533-1544

3. Morecroft I, Dempsie Y, Bader M, Walther DJ, Kotnik K, Loughlin L, Nilsen M, MacLean MR: Effect of tryptophan hydroxylase 1 deficiency on the development of hypoxia-induced pulmonary hypertension. $\mathrm{Hy}$ pertension 2007; 49(1):232-236.

4. Yu J, Feng HS, Chen BY, Qu P, Liu LB, Chen JK, Tie R, Huang XJ, Zhao $\mathrm{YF}, \mathrm{Zhu} \mathrm{XX}$ et al: Protective effects of vasonatrin peptide against hypobaric hypoxia-induced pulmonary hypertension in rats. Clin Exp Pharmacol Physiol 2010; 37(1):69-74.

5. Wang JR, Zhou Y, Sang K, Li MX: [Association between pulmonary vascular remodeling and expression of hypoxia-inducible factor-1alpha, endothelin-1 and inducible nitric oxide synthase in pulmonary vessels in neonatal rats with hypoxic pulmonary hypertension]. Zhongguo Dang Dai Er Ke Za Zhi 2013; 15(2):138-144.

6. Earley S, Resta TC: Estradiol attenuates hypoxia-induced pulmonary endothelin-1 gene expression. Am J Physiol Lung Cell Mol Physiol 2002; 283(1):L86-L93

7. Resta TC, Kanagy NL, Walker BR: Estradiol-induced attenuation of pulmonary hypertension is not associated with altered eNOS expression. Am J Physiol Lung Cell Mol Physiol 2001; 280(1):L88-L97.

8. Xu DQ, Luo Y, Liu Y, Wang J, Zhang B, Xu M, Wang YX, Dong HY, Dong $\mathrm{MQ}$, Zhao PT et al: Beta-estradiol attenuates hypoxic pulmonary hypertension by stabilizing the expression of p27kip1 in rats. Respir Res 2010; 11:182.

9. Lahm T, Albrecht M, Fisher AJ, Selej M, Patel NG, Brown JA, Justice MJ, Brown MB, Van Demark M, Trulock KM et al: 17beta-Estradiol attenuates hypoxic pulmonary hypertension via estrogen receptor-mediated effects. Am J Respir Crit Care Med 2012; 185(9):965-980.

10. Funakoshi T, Yanai A, Shinoda K, Kawano MM, Mizukami Y: G protein-coupled receptor 30 is an estrogen receptor in the plasma membrane. Biochem Biophys Res Commun 2006; 346(3):904-910.

11. Filardo EJ, Quinn JA, Bland KI, Frackelton AJ: Estrogen-induced activation of Erk-1 and Erk-2 requires the G protein-coupled receptor homo$\log$, GPR30, and occurs via trans-activation of the epidermal growth factor receptor through release of HB-EGF. Mol Endocrinol 2000; 14(10):1649-1660.

12. Harris CW, Shields JL, Hannon JP: Acute altitude sickness in females. Aerosp Med 1966; 37(11):1163-1167.

13. JHSA E: Cardiorepiratory studies in chronic mountain sickness (Monge's Syndrome). Respiration. 1971;: 485-517.
14. Ge RL, Helun G: Current concept of chronic mountain sickness: pulmonary hypertension-related high-altitude heart disease. Wilderness Environ Med 2001; 12(3):190-194.

15. Lahm T, Patel KM, Crisostomo PR, Markel TA, Wang M, Herring C, Meldrum DR: Endogenous estrogen attenuates pulmonary artery vasoreactivity and acute hypoxic pulmonary vasoconstriction: the effects of sex and menstrual cycle. Am J Physiol Endocrinol Metab 2007; 293(3):E865-E871.

16. Ji Y, Tang B, Traub RJ: The visceromotor response to colorectal distention fluctuates with the estrous cycle in rats. Neuroscience 2008; 154(4):1562-1567.

17. Cauley JA, Gutai JP, Glynn NW, Paternostro-Bayles M, Cottington E, Kuller LH: Serum estrone concentrations and coronary artery disease in postmenopausal women. Arterioscler Thromb 1994; 14(1):14-18.

18. Barrett-Connor E, Goodman-Gruen D: Prospective study of endogenous sex hormones and fatal cardiovascular disease in postmenopausal women. BMJ 1995; 311(7014):1193-1196.

19. Rexrode KM, Manson JE, Lee IM, Ridker PM, Sluss PM, Cook NR, Buring JE: Sex hormone levels and risk of cardiovascular events in postmenopausal women. Circulation 2003; 108(14):1688-1693.

20. Guthrie JR, Taffe JR, Lehert P, Burger HG, Dennerstein L: Association between hormonal changes at menopause and the risk of a coronary event: a longitudinal study. Menopause 2004; 11(3):315-322.

21. Lee JS, Yaffe K, Lui LY, Cauley J, Taylor B, Browner W, Cummings S: Prospective study of endogenous circulating estradiol and risk of stroke in older women. Arch Neurol 2010; 67(2):195-201.

22. Parker TA, Ivy DD, Galan HL, Grover TR, Kinsella JP, Abman SH: Estradiol improves pulmonary hemodynamics and vascular remodeling in perinatal pulmonary hypertension. Am J Physiol Lung Cell Mol Physiol 2000; 278(2):L374-L381.

23. Farhat MY, Chen MF, Bhatti T, Iqbal A, Cathapermal S, Ramwell PW: Protection by oestradiol against the development of cardiovascular changes associated with monocrotaline pulmonary hypertension in rats. Br J Pharmacol 1993; 110(2):719-723.

24. Rabinovitch M, Gamble WJ, Miettinen OS, Reid L: Age and sex influence on pulmonary hypertension of chronic hypoxia and on recovery. Am J Physiol 1981; 240(1):H62-H72.

25. Ou LC, Sardella GL, Leiter JC, Brinck-Johnsen T, Smith RP: Role of sex hormones in development of chronic mountain sickness in rats. J Appl Physiol 1994; 77(1):427-433.

26. Patel KM, Lahm T, Crisostomo PR, Herring C, Markel T, Wang M, Meldrum DR: The effects of endogenous sex hormones and acute hypoxia on vasoconstriction in isolated rat pulmonary artery rings. J Surg Res 2008; 146(1):121-126

27. Dubey RK, Gillespie DG, Mi Z, Rosselli M, Keller PJ, Jackson EK: Estradiol inhibits smooth muscle cell growth in part by activating the cAMP-adenosine pathway. Hypertension 2000; 35(1 Pt 2):262-266.

28. Dubey RK, Jackson EK, Gillespie DG, Zacharia LC, Imthurn B, Keller PJ: Clinically used estrogens differentially inhibit human aortic smooth muscle cell growth and mitogen-activated protein kinase activity. Arterioscler Thromb Vasc Biol 2000; 20(4):964-972.

29. Lindsey SH, Cohen JA, Brosnihan KB, Gallagher PE, Chappell MC: Chronic treatment with the $G$ protein-coupled receptor 30 agonist G-1 decreases blood pressure in ovariectomized mRen2.Lewis rats. Endocrinology 2009; 150(8):3753-3758.

30. Lindsey SH, Carver KA, Prossnitz ER, Chappell MC: Vasodilation in response to the GPR30 agonist G-1 is not different from estradiol in the mRen2.Lewis female rat. J Cardiovasc Pharmacol 2011; 57(5):598-603.

31. Christodoulakos GE, Lambrinoudaki IV, Botsis DC: The cardiovascular effects of selective estrogen receptor modulators. Ann N Y Acad Sci 2006; 1092:374-384

32. Petitti DB, Sidney S, Quesenberry CJ, Bernstein A: Incidence of stroke and myocardial infarction in women of reproductive age. Stroke 1997; 28(2):280-283

33. MacGregor EA, Frith A, Ellis J, Aspinall L, Hackshaw A: Incidence of migraine relative to menstrual cycle phases of rising and falling estrogen. Neurology 2006; 67(12):2154-2158.

34. Tostes RC, Nigro D, Fortes ZB, Carvalho MH: Effects of estrogen on the vascular system. Braz J Med Biol Res 2003; 36(9):1143-1158.

35. Stenmark KR, Fagan KA, Frid MG: Hypoxia-induced pulmonary vascular remodeling: cellular and molecular mechanisms. Circ Res 2006; 99(7):675-691.

36. Anderson GL, Limacher M, Assaf AR, Bassford T, Beresford SA, Black H, Bonds D, Brunner R, Brzyski R, Caan B et al: Effects of conjugated equine estrogen in postmenopausal women with hysterectomy: the Women's Health Initiative randomized controlled trial. JAMA 2004; 291(14):1701-1712. 
37. Doren M: Association between hormone replacement therapy and subsequent arterial and venous vascular events: a meta-analysis. Eur Heart $J$ 2009; 30(7):866-867.

38. Morrell ED, Tsai BM, Crisostomo PR, Wang M, Markel TA, Lillemoe KD, Meldrum DR: Therapeutic concepts for hypoxic pulmonary vasoconstriction involving ion regulation and the smooth muscle contractile apparatus. J Mol Cell Cardiol 2006; 40(6):751-760. 